PROCEEDINGS OF THE

AMERICAN MATHEMATICAL SOCIETY

Volume 130, Number 7 , Pages 2079-2088

S 0002-9939(02)06310-

Article electronically published on January 17, 2002

\title{
ON THE DIFFERENTIABILITY OF FIRST INTEGRALS OF TWO DIMENSIONAL FLOWS
}

\author{
WEIGU LI, JAUME LLIBRE, MARCEL NICOLAU, AND XIANG ZHANG
}

(Communicated by Carmen C. Chicone)

\begin{abstract}
By using techniques of differential geometry we answer the following open problem proposed by Chavarriga, Giacomini, Giné, and Llibre in 1999. For a given two dimensional flow, what is the maximal order of differentiability of a first integral on a canonical region in function of the order of differentiability of the flow? Moreover, we prove that for every planar polynomial differential system there exist finitely many invariant curves and singular points $\gamma_{i}, i=1,2, \cdots, l$, such that $\mathbb{R}^{2} \backslash\left(\bigcup_{i=1}^{l} \gamma_{i}\right)$ has finitely many connected open components, and that on each of these connected sets the system has an analytic first integral. For a homogeneous polynomial differential system in $\mathbb{R}^{3}$, there exist finitely many invariant straight lines and invariant conical surfaces such that their complement in $\mathbb{R}^{3}$ is the union of finitely many open connected components, and that on each of these connected open components the system has an analytic first integral.
\end{abstract}

\section{INTRODUCTION AND STATEMENT OF THE MAIN RESULTS}

Let

$$
\mathbf{X}=P \frac{\partial}{\partial x}+Q \frac{\partial}{\partial y}
$$

be a $C^{r}$ vector field with $r \geq 1$, defined on a two dimensional manifold $M$. Here, $r \geq 1$ means that $r=1,2, \cdots, \infty$, or $\omega$. Of course, when $r=\omega$, the flow is analytic. In this paper a two dimensional manifold means a two dimensional differentiable manifold (of class $C^{\infty}$ or of class $C^{\omega}$ in case the vector field $\mathbf{X}$ is assumed to be analytic), connected and without boundary, but not necessarily compact or orientable.

A map $\phi: D \rightarrow M$, or simply $(M, \phi)$, is the $C^{r}$ local flow with $r \geq 1$ associated to a vector field $\mathbf{X}$, with $D=\left\{(t, p): p \in M, t \in I_{p}\right\}$ and $I_{p}$ is the maximum open interval of the real line where the flow $\phi(t, p)=\phi_{p}(t)$ of $\mathbf{X}$ passing through $p \in M$ when $t=0$ is defined, if it verifies $d \phi_{p}(t) / d t=\mathbf{X}\left(\phi_{p}(t)\right)$, and

(i) $\phi(0, p)=p$ for all $p \in M$;

(ii) $\phi(t, \phi(s, p))=\phi(t+s, p)$ for all $p \in M$, and all $s$ and $t$ such that $s, t+s \in I_{p}$;

(iii) $\phi_{p}(-t)=\phi_{p}^{-1}(t)$ for all $p \in M$ such that $t,-t \in I_{p}$.

Received by the editors August 4, 2000 and, in revised form, February 16, 2001.

2000 Mathematics Subject Classification. Primary 34C05, 34C40, 37C10.

Key words and phrases. Two dimensional flows, polynomial systems, first integrals, differentiability. 
Two flows $(M, \phi)$ and $\left(M^{\prime}, \phi^{\prime}\right)$ are $C^{k}$ equivalent with $1 \leq k \leq r$ if there is a $C^{k}$ diffeomorphism of $M$ onto $M^{\prime}$ which takes orbits of $\phi$ onto orbits of $\phi^{\prime}$, preserving or reversing simultaneously the sense of all orbits.

Let $\phi$ be a $C^{r}$ local flow on the two dimensional manifold $M$ for $r \geq 1$. The flow $(M, \phi)$ is $C^{k}$ parallel if it is $C^{k}$-equivalent, with $1 \leq k \leq r$, to either the strip, the annular, the spiral or radial, or the toral flow. More precisely, these flows are respectively:

- $\left(\mathbb{R}^{2}, \phi\right)$ with the flow $\phi$ defined by $x^{\prime}=1, y^{\prime}=0$;

- $\left(\mathbb{R}^{2} \backslash\{0\}, \phi\right)$ with the flow $\phi$ defined (in polar coordinates) by $r^{\prime}=0, \theta^{\prime}=1$;

- $\left(\mathbb{R}^{2} \backslash\{0\}, \phi\right)$ with the flow $\phi$ defined by $r^{\prime}=r, \theta^{\prime}=0$ (i.e. this flow is called spiral or radial because in $\mathbb{R} \backslash\{0\}$ the flows $r^{\prime}=r, \theta^{\prime}=1$ and $r^{\prime}=r, \theta^{\prime}=0$ are equivalent).

- $\left(\mathbb{S}^{1} \times \mathbb{S}^{1}, \phi\right)$ with rational flow $\phi$ (i.e. the flow $x^{\prime}=1, y^{\prime}=0$ over the usual covering space $\mathbb{R}^{2}$ with rational slope; note in particular that all rational flows on the torus are equivalent).

The definition of parallel is given in [11. We note that Markus [10] gave the notion of $C^{0}$ parallel for the collection of curves filling a plane region of a planar $C^{0}$ vector field.

Let $p \in M$. We denote by $\gamma(p)$ the orbit of the flow $(M, \phi)$ passing through $p$ (i.e. $\gamma(p)=\left\{\phi(t, p): t \in I_{p}\right\}$ ), and by $\gamma^{+}(p)$ (respectively $\gamma^{-}(p)$ ) the positive semiorbit (respectively negative semiorbit), i.e. $\gamma^{+}(p)=\left\{\phi(t, p): t \in I_{p}\right.$ and $\left.t \geq 0\right\}$ (respectively $\gamma^{-}(p)=\left\{\phi(t, p): t \in I_{p}\right.$ and $\left.t \leq 0\right\}$ ).

We define by

$$
\alpha(p)=\operatorname{cl}\left(\gamma^{-}(p)\right)-\gamma^{-}(p) \quad\left(\text { respectively } \omega(p)=\operatorname{cl}\left(\gamma^{+}(p)\right)-\gamma^{+}(p)\right)
$$

the $\alpha$-limit of $\gamma^{-}(p)$ (respectively $\omega$-limit of $\gamma^{+}(p)$ ). Here, $\operatorname{cl}(\mathrm{A})$ denotes the closure of the subset $A$ of $M$. We note that these definitions of $\alpha$ - and $\omega$-limit sets do not coincide with the classical ones (see Hirsch and Smale [6]), but they are good for defining the notion of separatrix. Thus, the differences are that now the $\alpha$ - and $\omega$-limit sets of a singular point or a periodic orbit are the empty set.

An open neighbourhood $U$ of an orbit $\gamma(p)$ of the $C^{r}$ flow $(M, \phi)$ is said to be a $C^{k}$ parallel neighbourhood with $1 \leq k \leq r$ if $(U, \phi)$ is $C^{k}$ equivalent to a parallel flow for some $k \geq 1$.

We define the notion of separatrix following Neumann 11, but we remark that Markus [10] gave this notion of separatrix in the plane. An orbit $\gamma(p)$ is a separatrix of the flow $(M, \phi)$ if it is not contained in a parallel neighbourhood $(U, \phi)$ satisfying the following two assumptions:

(a) for any $q \in U, \alpha(q)=\alpha(p)$ and $\omega(q)=\omega(p)$;

(b) $\operatorname{cl}(U) \backslash U$ consists of $\alpha(p), \omega(p)$ and exactly two orbits $\gamma(a), \gamma(b)$ of $\phi$ with $\alpha(a)=\alpha(p)=\alpha(b)$ and $\omega(a)=\omega(p)=\omega(b)$.

With this definition of separatrix it is easy to check that any singular point, any limit cycle and any local separatrix of a hyperbolic sector are separatrices. We remark that an orbit of an irrational flow on the torus is not a separatrix.

We denote by $\Sigma$ the union of all separatrices of the flow $(M, \phi)$. Then $\Sigma$ is a closed invariant subset of $M$. Every connected component of the complement of $\Sigma$ in $M$, with the restricted flow, is called a canonical region of $\phi$.

We say that a $C^{k}$ function $H: M \backslash \Sigma \rightarrow \mathbb{R}$ with $k \geq 0$ is a weak first integral of the vector field $\mathbf{X}$ if $H$ is constant on every orbit of the flow $(M, \phi)$ defined by the 
vector field $\mathbf{X}$ located in every canonical region, and $H$ is nonconstant on any open subset of $M \backslash \Sigma$. As usual, a $C^{0}$ function means a continuous function. If $k \geq 1$, this definition is equivalent to

$$
\mathbf{X} H=\frac{\partial H}{\partial x} P+\frac{\partial H}{\partial y} Q \equiv 0
$$

on $M \backslash \Sigma$. Moreover, if $\Sigma=\emptyset$, then $H$ is called a first integral.

In [3] the authors obtained the following result.

Theorem 1. Every local flow $\phi$ on a two dimensional manifold $M$ has continuous first integrals on every canonical region of $\phi$.

Meanwhile, they proposed the following:

Open problem. What is the maximal order of differentiability of the first integrals on a canonical region of a given two dimensional flow $\phi$ in function of the order of differentiability of the flow?

In this paper we answer the open problem. The results are the following.

Theorem 2. Let $\phi$ be a $C^{r}$ flow on a two dimensional manifold $M$ with $r \geq 1$, and let $\Sigma$ be the union of all separatrices of $\phi$. Then:

(1) Every canonical region of $(M, \phi)$ is $C^{r}$ parallel.

(2) The flow $\phi$ restricted to every canonical region has a $C^{r}$ (respectively $C^{\infty}$, $C^{\omega}$ ) first integral for $r \in \mathbb{N}$ (respectively $r=\infty, \omega$ ).

As usual $\mathbb{N}$ denotes the set of positive integers. Statement (1) in the case $C^{0}$ parallel was proved by Neumann [11]; see Section 2.

We consider planar differential systems

$$
\frac{d x}{d t}=\dot{x}=P(x, y), \quad \frac{d y}{d t}=\dot{y}=Q(x, y),
$$

where $P$ and $Q$ are $C^{r}$ functions for $r \geq 0$ defined in an open subset $U$ of $\mathbb{R}^{2}$. If $P$ and $Q$ are polynomials in the variables $x$ and $y$, we say that (1) is a polynomial system.

In the next two theorems we say that any analytic vector field on $\mathbb{S}^{2}$ has finitely many limit cycles as was proved by Il'Yashenko [8] and Ecalle [5]. The following results improve Theorem 2 for planar polynomial differential systems.

Theorem 3. For every planar polynomial system there exist finitely many invariant curves in $\mathbb{R}^{2}$ and singular points $\gamma_{i}, i=1,2, \cdots, l$, such that $\mathbb{R}^{2} \backslash\left(\bigcup_{i=1}^{l} \gamma_{i}\right)$ has finitely many connected open sets, and on each of these connected sets the system has an analytic first integral.

Corollary 4. Let $\mathbf{X}=(P, Q)$ be a polynomial vector field in $\mathbb{R}^{2}$ such that $P$ and $Q$ are coprime. Then, using the notations of Theorem 3, the set $\bigcup_{i=1}^{l} \gamma_{i}$ is formed by all the separatrices of $\mathbf{X}$ in $\mathbb{R}^{2}$, and the open components of $\mathbb{R}^{2} \backslash\left(\bigcup_{i=1}^{l} \gamma_{i}\right)$ are the canonical regions of $\mathbf{X}$. 3

The proof of Corollary 4 can be easily obtained by following the proof of Theorem

Next we consider the homogeneous polynomial vector field

$$
\mathbf{Q}(\mathbf{x})=\left(Q_{1}(\mathbf{x}), Q_{2}(\mathbf{x}), Q_{3}(\mathbf{x})\right), \quad \mathbf{x}=\left(x_{1}, x_{2}, x_{3}\right) \in \mathbb{R}^{3} .
$$

We obtain the following results related with its first integrals. 
Theorem 5. For a homogeneous polynomial vector field $\mathbf{Q}(\boldsymbol{x})$ in $\mathbb{R}^{3}$ having each component of the same degree, there exist finitely many invariant straights lines and invariant conical surfaces such that their complement in $\mathbb{R}^{3}$ is the union of finitely many invariant open connected components, and on each of these connected open components the vector field has an analytic first integral.

This paper is organized as follows. In Section 2, we prove Theorem[2, In Sections 3 and 4 we prove Theorems 3 and 5 respectively.

\section{Proof of Theorem 2}

The following result due to Neumann [11 plays a main role in the proof of our Theorem 2 since its proof is short, we give it here.

Lemma 6. The flow of $(M, \phi)$ on every canonical region is a $C^{0}$-parallel flow given by either a strip, an annular, a spiral, or a toral flow.

Proof. Let $U$ be a canonical region of the $C^{0}$ flow $(M, \phi)$. We denote the flow $\phi$ on $U$ by $\left(U, \phi^{\prime}=\left.\phi\right|_{U}\right)$. Since there are no separatrices in $U$, the set consisting of orbits homeomorphic with $\mathbb{S}^{1}$ is open, and similarly the set consisting of orbits homeomophic with $\mathbb{R}$ is open. Hence $U$ consists entirely of closed orbits or entirely of line orbits.

We claim that two orbits of $\phi^{\prime}$ can be separated with disjoint parallel neighborhoods. To prove this, we suppose that $\gamma(p)$ and $\gamma(q)$ are distinct orbits (closed or not) which cannot be separated. Then, for any parallel neighborhood $N_{p}$ of $p$, we have $q \in \operatorname{cl}\left(N_{p}\right)$; i.e., $q \in \bigcap_{N_{p}} \operatorname{cl}\left(N_{p}\right)=\alpha(p) \cup \gamma(p) \cup \omega(p)$. This means that $q \in \alpha(p)$ (or $q \in \omega(p)$ ), but it is impossible because $q \in N_{q} \subset U$ and $\alpha(p) \cup \omega(p) \subset \operatorname{cl}\left(N_{q}\right) \backslash N_{q} \not \subset U$.

It follows that the quotient space $U / \phi^{\prime}$, obtained by collapsing orbits of $\left(U, \phi^{\prime}\right)$ to points, is a (Hausdorff) one dimensional manifold. Hence the natural projection $\pi: U \rightarrow U / \phi^{\prime}$ is a locally trivial fibering; there are only four possibilities, the four classes of parallel flows described above. This proves the lemma.

Proof of Theorem Q Let $M$ be a two dimensional differentiable manifold and $\phi$ be a $C^{r}$ flow defined on it by the vector field $\mathbf{X}$. If the manifold $M$ is compact, the flow $\phi$ is complete; i.e. for every $q \in M, \phi_{q}(t)=\phi(t, q)$ is defined for all $t \in \mathbb{R}$.

In general the manifold $M$ is not compact and the flow need not be complete. We will see that the restriction of the flow to a given non-compact canonical region $U$ can be rescaled in such a way that it becomes complete. The proof uses the existence of a Riemannian metric on $U$ which is geodesically complete and of the same class of differentiability $\left(C^{\infty}\right.$ or $\left.C^{\omega}\right)$ as the manifold $M$. In case the manifold is of class $C^{\infty}$ the metric can be constructed easily as follows. Let $g$ be an arbitrary Riemannian metric on $U$ of class $C^{\infty}$. It is well known (see [15]) that there is an increasing sequence of compact subsets $K_{m}$ exhausting $U$. That is, an infinite sequence of compact subsets $K_{m} \subset U$ such that $K_{m}$ is contained in the interior of $K_{m+1}$ and that $U=\bigcup K_{m}$. Then one can construct a $C^{\infty}$ function $\sigma$ on $U$ such that the new Riemannian metric $g^{\prime}=e^{\sigma} g$ has the following property: the distance (with respect to $g^{\prime}$ ) between $K_{m}$ and $\overline{U-K_{m+1}}$ is bigger than one. Then it follows from the theorem of Hopf and Rinow (see [4]) that $g^{\prime}$ is geodesically complete. 
The analytic case is much more involved. We recall that the differentiable structure of a smooth manifold $N$ underlies a real analytic structure. This follows from Whitney's embedding theorem [16] which states that, for a given $n$-dimensional differentiable manifold $N$, there is a smooth proper embedding $f: N \rightarrow \mathbb{R}^{2 n+1}$ such that $f(N)$ is an analytic submanifold of $\mathbb{R}^{2 n+1}$. If $\mathcal{D}$ denotes the maximal $C^{\infty}$ atlas defining the differentiable structure of $N$, then $f$ induces on $N$ an atlas $\mathcal{A}_{W}$ contained in $\mathcal{D}$ and such that the coordinate changes are analytic. We consider on $N \equiv f(N)$ the Riemannian metric $g$ induced by the euclidean metric of $\mathbb{R}^{2 n+1}$. Clearly it is analytic and we claim that it is also geodesically complete. Let $D_{n}$ denote the metric closed ball (with respect to $g$ ) on $N \equiv f(N)$ centered at a given point $p \in f(N)$ and of radius $n \in \mathbb{N}$. It also follows from Hopf-Rinow's theorem that $g$ is complete if $D_{n}$ is a compact subset of $N$ for all $n \in \mathbb{N}$. Let $B_{n}$ be the euclidean closed ball in $\mathbb{R}^{2 n+1}$ centered at $p$ and of radius $n \in \mathbb{N}$. Since the distance associated to the metric $g$ between two points in $f(N)$ is bigger than its euclidean distance we have $D_{n} \subset\left(B_{n} \cap f(N)\right)$. The fact that the map $f$ is proper then implies that the sets $B_{n} \cap f(N)$ are compact and therefore $D_{n}$ are compact, too. This proves the claim.

We now use the fact that two different analytic structures on a manifold underlying a given $C^{\infty}$ structure are isomorphic. This was proved in the compact case by Royden [13] and for open non-compact manifolds by Huebsch and Morse 7]. In our situation this means that, if $\mathcal{D}$ and $\mathcal{A}$ denote the maximal atlases of the canonical region $U$ defining respectively its differentiable and its analytic structure, and if $\mathcal{A}_{W}$ is the analytic atlas given by Whitney's embedding, then there is a $C^{\infty}$ diffeomorphism $h: U \rightarrow U$ such that $h^{*} \mathcal{A}_{W}=\mathcal{A}$, i.e. $\tau \circ h$ is a local chart in $\mathcal{A}$ for each local chart $\tau$ in $\mathcal{A}_{W}$. Then $h^{*} g$ is a Riemannian metric on $U$ which is complete and analytic with respect to the original analytic structure defined by the atlas $\mathcal{A}$.

We remark that, since we are dealing with two dimensional manifolds, the existence of the complete Riemannian metric in the analytic case could also be proved using the Riemann uniformization theorem instead of Whitney's theorem.

Now we claim that the vector field $\mathbf{Y}=\mathbf{X} /\|\mathbf{X}\|$ on $U$, where $\|\cdot\|$ stands for the norm associated to the metric constructed above, is complete and of class $C^{r}$, where $r=1, \ldots, \infty, \omega$ is the degree of differentiability of $\mathbf{X}$.

Let $\psi$ denote the flow on $U$ associated to $\mathbf{Y}$. It follows from the above lemma that the positive (respectively negative) semiorbit of $\psi$ through a given point $p \in U$ is closed, and therefore $\psi(t, p)$ is defined for each time, or is not contained in any compact subset of $U$. Since the metric is complete in the second situation the semiorbit will have an infinite length. But by construction the time parameter of $\psi$ is just the arc-length of the orbit, hence $\psi(t, p)$ is also defined for each time. This proves the completeness of $\psi$.

Let $(U, \psi)$ be a canonical region of $M$ where $\psi$ denotes either the complete $C^{r}$ flow constructed above in the case $U$ is noncompact or the original flow $\phi$ in the case $U$ is compact. For $p \in U$ let $\Sigma_{p}$ be a local transversal section of the flow $\psi$ with $p \in \Sigma_{p}$. Let $V_{p}$ be the set $\left\{\psi(t, q) \in U: t \in \mathbb{R}\right.$ and $\left.q \in \Sigma_{p}\right\}$. Then if the canonical region $(U, \psi)$ is a strip or a spiral, respectively an annular or a toral, the flow $\psi$ defines a $C^{r}$ diffeomorphism:

$$
\begin{aligned}
\eta_{p}^{-1}=\psi: \quad \Sigma_{p} \times \mathbb{R} & \longrightarrow V_{p}, \\
(q, t) & \longrightarrow \psi(t, q),
\end{aligned}
$$


respectively

$$
\begin{aligned}
\eta_{p}^{-1}=\psi: \quad \Sigma_{p} \times \mathbb{S}^{1} & \longrightarrow V_{p}, \\
(q, t) & \longrightarrow \psi(t T(q), q),
\end{aligned}
$$

where $T(q)$ denotes the minimal period for which the flow $\psi$ with initial point $q$ moves along $\mathbb{S}^{1}$. Moreover, in both cases the inverse map $\eta_{p}$ is also a $C^{r}$ diffeomorphism.

Take $p, q \in U$ such that $V_{p} \cap V_{q} \neq \emptyset$. We have that the map $\eta_{q} \circ \eta_{p}^{-1}$ is a $C^{r}$ diffeomophism. This proves that the quotient space $U / \psi$ has a natural structure of $C^{r}$ manifold of dimension 1 which is $C^{r}$ equivalent to either $\mathbb{R}$ or $\mathbb{S}^{1}$. Moreover the projection $U \rightarrow U / \psi$ is a locally trivial fibre bundle of class $C^{r}$ with fibre $\mathbb{R}$ or $\mathbb{S}^{1}$. Lemma 6 implies that this fibre bundle is trivial and, according to the different possibilities, the flow $(U, \psi)$ is $C^{r}$ equivalent to either the strip, annular, spiral or the toral flow. This proves that the canonical region $U$ of $M$ is $C^{r}$ parallel.

We now prove statement (2). From statement (1) it follows that for the $C^{r}$ flow $\psi$ associated with the vector field $\mathbf{Y}$, in every canonical region $U$ of $M$ there exists a $C^{r}$ diffeomorphism $h$ from $(U, \psi)$ onto $(V, \xi)$ which takes orbits of $\psi$ onto orbits of $\xi$ preserving or reversing simultaneously the sense of all orbits, where $(V, \xi)$ is one of the four parallel flows. For the strip and toral flows the first integral is $H(x, y)=y$, for the annular flow the first integral is $H(r, \theta)=r$, and for the spiral flow the first integral is $H(r, \theta)=\theta$. Moreover, we obtain that $h^{-1} \circ H$ is a $C^{r}$ first integral of the $C^{r}$ flow $\psi$ on the canonical region $U$. This proves the theorem.

\section{Some Preliminaries And the proof of Theorem 3}

We first recall some basic results, which will be used later on.

Poincaré-Bendixson Theorem. (See Anosov and Arnold [1] or Roussarie [12], Chapter 1.) Let $\mathbf{X}$ be a vector field on a compact surface $S$ of genus 0 . Suppose that every singular point of $\mathbf{X}$ is isolated. Then the $\omega$-limit set of the phase curves of $\mathbf{X}$ must be one of the following types: a singular point; a periodic orbit; or a polycycle, i.e. a union of finitely many singular points $p_{1}, \cdots, p_{m}$, and finitely many orbits $\gamma_{1}, \cdots, \gamma_{m}$ such that $\alpha\left(\gamma_{i}\right)=p_{i}$ for $i=1, \cdots, n$ and $\beta\left(\gamma_{i}\right)=p_{i+1}$ for $i=1, \cdots, m-1$ and $\beta\left(\gamma_{m}\right)=p_{1}$. Eventually some of the points $p_{i}$ can be identified.

Theorem 7 (see Il'Yashenko 8, or Lefschetz 9] Chapter X). Any isolated singular point of a two dimensional analytic system can only be one of following three cases: a center; a focus; a singular point whose neighbourhood is a union of finitely many hyperbolic, elliptic or parabolic sectors.

The Bendixson compactified vector field $b(\mathbf{X})$ corresponding to a planar polynomial vector field $\mathbf{X}$ is an analytic vector field induced on $\mathbb{S}^{2}$ by the stereographic projection. The sphere in $\mathbb{R}^{3}$ given by $x^{2}+y^{2}+z^{2}=1$ is called the Bendixson sphere. The compactified vector field $b(\mathbf{X})$ has the north pole $N=(0,0,1)$ on $\mathbb{S}^{2}$ as a singular point. For more details, see for instance [14. We note that the flow given by the compactified vector field $b(\mathbf{X})$ in $\mathbb{S}^{2} \backslash\{N\}$ is topologically equivalent with the flow given by $\mathbf{X}$ in $\mathbb{R}^{2}$, and that $N$ corresponds to the infinity of the planar polynomial vector field $\mathbf{X}$.

Proposition 8. Assume that $P$ and $Q$ are polynomials in the variables $x$ and $y$, and that they are relatively prime. Then the compactified vector field $b(\mathbf{X})$ associated 
with the vector field $\mathbf{X}=(P, Q)$ has finitely many separatrices and canonical regions in the Bendixson sphere.

Proof. From the assumptions of the proposition and the Bendixson compactification we know that the compactified vector field $b(\mathbf{X})$ has finitely many singular points on $\mathbb{S}^{2}$ (the finitely many finite singular points and the unique one at infinity). So they are isolated. Since the compactified vector field $b(\mathbf{X})$ is $C^{\omega}$ on $\mathbb{S}^{2}$, by Theorem 7 we get that its singular points are centers, foci, or union of finitely many hyperbolic, elliptic or parabolic sectors.

Again as the compactified vector field $b(\mathbf{X})$ is $C^{\omega}$, from the Poincaré-Bendixson Theorem we obtain that on the Bendixson sphere the $\alpha$-limit and $\omega$-limit of every orbit for the compactified vector field $b(\mathbf{X})$ is a singular point (finite or infinite), a periodic orbit, or a polycycle.

We claim that each separatrix of the compactified vector field $b(\mathbf{X})$ is one of the following types: a singular point (finite or infinite); a limit cycle; or a boundary of a hyperbolic sector of a singular point.

We now prove the claim. By the definition of separatrix it is easy to prove that every singular point is a separatrix, and that each limit cycle is also a separatrix.

A boundary orbit $\gamma$ of a hyperbolic sector is a separatrix because there are no parallel neighbourhoods which contain $\gamma$ and satisfy the conditions (a) and (b). Otherwise, all orbits in the parallel neighborhood have the same $\alpha$-limit and the same $\omega$-limit, and this is in contradiction with the fact that the orbit $\gamma$ is in the boundary of a hyperbolic sector.

From the Poincaré-Bendixson Theorem and Theorem 7 it is easy to obtain that the separatrices of the compactified vector field $b(\mathbf{X})$ can only be singular points, limit cycles, or the boundary orbits of a hyperbolic sector. Here we use the fact that a spiral trajectory $\Gamma$ from a singular point to a limit cycle or to a separatrix loop is not a separatrix. Because for any $p \in \Gamma$ there exists a sufficiently small open transversal $L$ to the flow centered at $p$ such that the union of all the orbits starting at points of $L$ forms a parallel region satisfying (a) and (b) in the definition of separatrices. This proves the claim.

We recall that under our assumptions the compactified vector field $b(\mathbf{X})$ has finitely many singular points. Il'Yashenko 8 proved that a given analytic system on $\mathbb{S}^{2}$ has finitely many limit cycles (see also Ecalle [5]). Since the compactified vector field $b(\mathbf{X})$ is analytic, we get from Theorem 7 that the number of boundaries of hyperbolic sectors of all singular points (finite and infinite) for the compactified vector field $b(\mathbf{X})$ is finite. Therefore, the compactified vector field $b(\mathbf{X})$ has finitely many separatrices on the Bendixson sphere.

Since the boundary of every canonical region is formed by separatrices, the compactified vector field $b(\mathbf{X})$ has finitely many canonical regions on the Bendixson sphere. This completes the proof of the proposition.

Proof of Theorem 3 . If the polynomial system (1) has infinitely many singular points in the finite plane, then the polynomials $P$ and $Q$ must have a common real factor. Let $B(x, y)$ be the maximum common real factor of $P$ and $Q$, and $P=B \bar{P}(x, y)$ and $Q=B \bar{Q}(x, y)$. Then, from the definition of first integral it follows that system (1) and the system

$$
\dot{x}=\bar{P}(x, y), \quad \dot{y}=\bar{Q}(x, y),
$$


have the same first integrals. Obviously, the vector field $\overline{\mathbf{X}}=(\bar{P}, \bar{Q})$ has finitely many finite singular points.

From Proposition 8 the compactified vector field $b(\overline{\mathbf{X}})$ associated with $\overline{\mathbf{X}}$ has finitely many separatrices on $\mathbb{S}^{2}$, because the curves $\Gamma_{i}^{\prime} s$ intersect in finitely many points which are singular points. We denote by $\Gamma_{i}$ for $i=1,2, \cdots, l$ the separatrices of $b(\overline{\mathbf{X}})$. Then $\mathbb{S}^{2} \backslash\left(\bigcup_{i=1}^{l} \Gamma_{i}\right)$ is a union of finitely many connected open sets. Every connected open set is a canonical region. From Theorem 2 and the compactification of the vector fields on $\mathbb{S}^{2}$ we obtain that the vector field $b(\overline{\mathbf{X}})$ has an analytic first integral $H$ on every canonical region.

Every separatrix $\Gamma_{i}$ of $b(\overline{\mathbf{X}})$ on $\mathbb{S}^{2}$ corresponds to an invariant curve $\gamma_{i}$ of $\overline{\mathbf{X}}$ in $\mathbb{R}^{2}$ except infinity. Let $\Sigma^{\prime}$ be the union of such $\gamma_{i}$. Then $\mathbb{R}^{2} \backslash \Sigma^{\prime}$ is a finite union of the canonical regions of $\overline{\mathbf{X}}$ in $\mathbb{R}^{2}$. Since in the process of Bendixson compactification the projection $f$ from $\mathbb{R}^{2}$ to $\mathbb{S}^{2}$ is analytic, $H \circ f$ is an analytic first integral of $\overline{\mathbf{X}}$ in the corresponding canonical region $\Omega$. Consequently, $H \circ f$ is an analytic first integral of $\mathbf{X}$ in the connected open set $\Omega$. This proves the theorem.

\section{Proof of Theorem 5}

Consider the homogeneous polynomial vector field $\mathbf{Q}(\mathbf{x})=\left(Q_{1}(\mathbf{x}), Q_{2}(\mathbf{x}), Q_{3}(\mathbf{x})\right)$, where $Q_{i}(\mathbf{x})$ for $i=1,2,3$, are homogeneous polynomials in $\mathbf{x}=\left(x_{1}, x_{2}, x_{3}\right)$ of the same degree. Since the vectors defined by $\mathbf{Q}(\mathbf{x})$ are parallel at all points on the straight line passing through the origin $(0,0,0)$, we project all the vectors $\mathbf{Q}(\mathbf{x})$ along every straight line passing through the origin onto the sphere $\mathbb{S}^{2}$, and we get a tangent vector field $\mathbf{Q}_{T}(\mathbf{x})$ on $\mathbb{S}^{2}$. Obviously, we have

$$
\mathbf{Q}_{T}(\mathbf{x})=\mathbf{Q}(\mathbf{x})-\langle\mathbf{x}, \mathbf{Q}(\mathbf{x})\rangle \cdot \mathbf{x}, \quad \mathbf{x} \in \mathbb{S}^{2},
$$

where $\langle\cdot, \cdot\rangle$ denotes the inner product of two vectors and $\langle\mathbf{x}, \mathbf{Q}(\mathbf{x})\rangle \cdot \mathbf{x}$ denotes the projection of $\mathbf{Q}(\mathbf{x})$ on the direction $\mathbf{x}$ (see for instance, Camacho [2] and Ye [17, and $\S 22)$. Moreover, the vector field $\mathbf{Q}_{T}(\mathbf{x})$ is symmetric or anti-symmetric with respect to the origin.

Define $\Pi_{3}=\left\{\mathbf{x} \in \mathbb{R}^{3}: x_{3}=1\right\}$. Then $\Pi_{3}$ is the tangent plane to $\mathbf{S}^{2}$ at $(0,0,1)$. We now define a vector field in $\Pi_{3}$ as follows:

$$
\mathbf{W}_{Q}(\mathbf{x})=\left(W_{1}(\mathbf{x}), W_{2}(\mathbf{x}), 0\right)=\left(-x_{1} Q_{3}(\mathbf{x})+Q_{1}(\mathbf{x}),-x_{2} Q_{3}(\mathbf{x})+Q_{2}(\mathbf{x}), 0\right),
$$

where $\mathbf{x}=\left(x_{1}, x_{2}, 1\right)$. We note that the vector field $\mathbf{W}_{Q}$ is simply the central projection of the vector field $\mathbf{Q}_{T}$ on $\mathbb{S}^{2}$ to $\Pi_{3}$. It follows from Camacho [2] that the vector field $\mathbf{W}_{Q}(\mathbf{x})$ is tangent to the intersection curve of $\Omega(\gamma)=\{t p: t \in \mathbb{R}, p \in \gamma\}$ with $\Pi_{3}$, where $\gamma$ is a trajectory of $\mathbf{Q}_{T}(\mathbf{x})$ on $\mathbb{S}^{2}$. Then $\Omega(\gamma)$ is a surface constructed by straight lines connecting the origin with all points of $\gamma$. Furthermore, this surface is invariant under the flow of $\mathbf{Q}(x)$, and it is called an invariant conical surface. This conical surface is symmetric with respect to the origin.

It is easy to prove that for an arbitrary point $P \in \mathbb{S}^{2}$, if $P$ is a singular point of $\mathbf{Q}_{T}(\mathbf{x})$ and $\mathbf{Q}(\mathbf{x})$, then the straight line $l$ connecting $O$ and $P$ is formed by singular points of $\mathbf{Q}(\mathbf{x})$. If $P$ is a singular point of $\mathbf{Q}_{T}(\mathbf{x})$, but not a singular point of $\mathbf{Q}(\mathbf{x})$, then $l$ is an invariant straight line of $\mathbf{Q}(\mathbf{x})$ with a unique singular point $O$ on $l$.

If the vector field $W_{Q}(\mathbf{x})$ has infinitely many singular points, it must have common factors. By rescaling the time we eliminate the maximum common factor, then the new vector field through the central projection produces a vector field $\overline{\mathbf{P}}_{T}(\mathbf{x})$ on $\mathbb{S}^{2}$ with finitely many singular points outside the equator. If the vector field 
$\overline{\mathbf{P}}_{T}(\mathbf{x})$ has infinitely many singular points on the equator, working in a similar way to the proof of Theorem [3. we can get a vector field $\mathbf{P}_{T}(\mathbf{x})$ on $\mathbb{S}^{2}$ that has finitely many singular points on every local chart.

Using the arguments of the proofs of Theorems 2 and 3 we obtain that the vector field $\mathbf{P}_{T}(\mathbf{x})$ on $\mathbb{S}^{2}$ has finitely many canonical region on $\mathbb{S}^{2}$, and that in every canonical region the vector field $\mathbf{P}_{T}(\mathbf{x})$ has an analytical first integral.

We claim that the boundary of every canonical region of $\mathbf{P}_{T}(\mathbf{x})$ is invariant under the flow of $\mathbf{Q}_{T}(\mathbf{x})$, and that the first integral of $\mathbf{P}_{T}(\mathbf{x})$ in every canonical region is also a first integral of $\mathbf{Q}_{T}(\mathbf{x})$. The first statement follows easily. We now prove the second statement. For any first integral $H(\mathbf{x})$ and any point $\mathbf{p}$ in a canonical region $U$ of $\mathbf{P}_{T}(\mathbf{x})$, if $\mathbf{Q}_{T}(\mathbf{p}) \neq 0$, then the vector is parallel to the vector $\mathbf{P}_{T}(\mathbf{p}) \neq 0$, so along every orbit in $U$ different from a singular point of $\mathbf{Q}_{T}(\mathbf{x}) H(\mathbf{x})$ is also a constant. If $\mathbf{p}$ is a singular point of $\mathbf{Q}_{T}(\mathbf{x})$ in $U$, on this special orbit $H(\mathbf{x})$ is clearly a constant. Thus, we obtain an analytical first integral of $\mathbf{Q}_{T}(\mathbf{x})$ on each of finitely many open connected canonical regions of $\mathbb{S}^{2}$.

Since the boundaries of the canonical regions are invariant under the flow of $\mathbf{Q}_{T}(\mathbf{x})$, the surfaces constructed by the rays connecting $O$ and all the points on the boundaries form finitely many invariant straight lines and invariant conical surfaces. In $\mathbb{R}^{3}$ the complement of these invariant straight lines and conical surfaces are finitely many open connected conical tubes. If $H(\mathbf{x})$ is an analytic first integral of $\mathbf{Q}_{T}(\mathbf{x})$ in a canonical region, then $F(\mathbf{x})=H(\mathbf{x} /\|\mathbf{x}\|)$ is an analytic first integral of $\mathbf{Q}(\mathbf{x})$ in the corresponding conical tube, where $\|\cdot\|$ denotes the norm induced by the Euclidean distance. This completes the proof of the theorem.

\section{ACKNOWLEDGEMENTS}

The first author was partially supported by the Departament de Matemàtiques of the Universitat Autònoma de Barcelona, by NSFC of China and by the 973 Project of the Ministry of Science and Technology of China. The second author was partially supported by a DGES grant number PB96-1153 and by a CICYT grant number 1999SGR 00349. The third author was partially supported by a DGICYT grant number PB96-1178. The fourth author was partially supported by the Ministerio de Educaciøn y Cultura of Spain, grant number SB97-50922201, and by NSFC of China, grant number 19901013. The first and fourth authors express their thanks to the Centre de Recerca Matemática and to the Departament de Matemàtiques of the Universitat Autònoma de Barcelona for their hospitality and support during the period in which this paper was written.

\section{REFERENCES}

1. D. V. Anosov And V. I. Arnold, Dynamical Systems I, Springer-Verlag, Berlin Heidelberg, 1988. MR 89g:58060

2. M.I.T. CAMACHO, Geometric properties of homogeneous vector fields of degree two in $\mathbb{R}^{3}$, Trans. Amer. Math. Soc. 268 (1981), 79-101. MR 83b:58041

3. J. Chavarriga, H. Giacomini, J. Giné, And J. Llibre, On the integrability of twodimensional flows, J. Differential Equations 157 (1999), 163-182. MR 2000h:37058

4. M. P. Do CARMo, Riemannian geometry, Birkhäuser, Boston, 1992. MR 92i:53001

5. J. ECALLE, Introduction aux fonctions analysables et preuve constructive de la conjecture de Dulac, Hermann, Paris, 1992. MR 97f:58104

6. M.W. Hirsch and S. Smale, Differential equations, dynamical systems, and linear algebra, Pure and Applied Mathematics, Vol. 60, Academic Press, New York, 1974. MR 58:6484] 
7. W. Huebsch and M. Morse, Diffeomorphisms of manifolds, Rend. Circ. Mat. Palermo (2) 11 (1962), 291-318. MR 30:2516

8. Yu. S. IL'Yashenko, Finiteness Theorems for Limit Cycles, Transl. Math. Monographs 94, Amer. Math. Soc., Providence, R. I., 1991. MR 92k:58221

9. S. Lefschetz, Differential Equations: Geometric Theory, Dover Publications, Inc., New York, 1977. MR 55:8441

10. L. Markus, Global structure of ordinary differential equations in the plane, Trans. Amer. Math. Soc. 76 (1954), 127-148. MR 15:704a

11. D. A. Neumann, Classification of continuous flows on 2-manifolds, Proc. of Amer. Math. Soc. 48 (1975), 73-81. MR 50:8609

12. R. Roussarie, Bifurcation of Planar Vector Fields and Hilbert's Sixteenth Problem, Progress in Mathematics 164, Birkhauser Verlag, Basel, Switzerland, 1998. MR 99k:58129

13. H.L. Royden, The analytic approximation of differentiable mappings, Math. Ann. 139 (1960), 171-179. MR 22:4067]

14. S. Schecter And M. Singer, Planar polynomial foliations, Proc. Amer. Math. Soc. 79 (1980), 649-656. MR 82j:58096a

15. F.W. WARner, Foundations of differentiable manifolds and Lie groups, Scott, Foresman and Co., London, 1971. MR 45:4312

16. H. Whitney, Differentiable manifolds, Annals of Math. 37 (1936), 645-680.

17. Ye YAnqIan, Qualitative Theory of polynomial Differential Systems (in Chinese), Shanghai Scientific \& Technical Publishers, Shanghai, 1995.

Department of Mathematics, Peking University, Beijing 100871, People's Republic of CHINA

E-mail address: weigu@sxx0.math.pku.edu.cn

Departament de Matemàtiques, Universitat Autònoma de Barcelona, 08193 - BelLATERra, BArcelona, Spain

E-mail address: jllibre@mat.uab.es

Departament de Matemàtiques, Universitat Autònoma de Barcelona, 08193 - BelLATERra, BARCElona, Spain

E-mail address: nicolau@mat.uab.es

Department of Mathematics, Nanjing Normal University, Nanjing 210097, People's Republic of China

E-mail address: xzhang@pine.njnu.edu.cn 